\title{
Loop Quantum Effects on a Viscous Dark Energy Cosmological Model
}

\section{N.Mebarki ${ }^{1}$}

Laboratoire de Physique Mathematique et Subatomique, Mentouri University

Route Ain El Bey, Constantine 25000, Algeria

E-mail: nnmebarki@yahoo.fr

\section{S.Benchick}

Laboratoire de Physique Mathematique et Subatomique, Mentouri University

Route Ain El Bey, Constantine 25000, Algeria

E-mail: benchikhsara@gmail.com

A novel effective cosmological model with bulk viscosity and loop quantum geometry effects is proposed. It is found that the bulk viscosity affects the quintessence scenario leading to the existence of a De Sitter type viscous late time attractor whereas the loop quantum effects influence the phantom case where the big rip singularity is removed.

Frontiers of Fundamental Physics 14

FFP14

15-18 July 2014

Aix Marseille University (AMU) Saint-Charles Campus, Marseille

$1 \quad$ Speaker 


\section{Introduction}

One of the greatest challenges in modern cosmology is the attempt to understand the nature and the origin of the present acceleration expansion of our universe $[1,2,3,4]$, which has been confirmed by several recent observations such asType Ia Supernovae (SNeIa) [5], cosmic microwave background (CMB) anisotropy[6] and large scale structure (LSS) [7]. Confirmed observations indicates that dark energy (DE) dominates our present universe and the viability of the bulk viscous dark energy scenario to take part in explaining the presently accepted cosmological paradigm is justified [8]. Moreover, it is believed that quantum gravity effects may play an important role in the evolution of the universe. Thus, it is preferred to study the properties of cosmological models in the framework of a quantum gravity theory. In this paper, we work with a Loop Quantum Cosmology (LQC) effective model, which is a canonical quantization of an homogeneous space-time based on a reduced symmetry model of loop quantum gravity $[9,10,11]$. In fact, the effects of loop quantum gravity consist of modifying the standard Friedmann equations by adding a correction term of the form $-\rho^{2} / \rho_{c}$ encoding the discrete quantum geometric nature of the space time. When this correction term becomes dominant, the universe starts to bounce and then expands backwards. One of the major successes in LQC is that the big bang singularity can be replaced by a big bounce and future singularities can be also avoided [12,13].The goal of this paper is to combine both loop quantum cosmology effects and bulk viscous dark energy in one model trying to find more hints behind cosmic observations. Our paper is organized as follows: In section 2, we present the dynamical behavior of a universe filled with pressureless dark matter and viscous scalar field within the standard classical cosmology (SC). Then, we extend our study by taking into account the LQC effects and finally, in section 3, we draw our conclusions.

\section{Formalism}

In SC, the first Friedmann equation is given by

$$
H^{2}=\left(\rho_{D M}+\rho_{D E}\right) / 3
$$

and in the presence of a constant bulk viscosity, the modified Raychaudhuri equation reads:

$$
\dot{H}=-\frac{1}{2}\left(\rho_{D M}+(1+\omega) \rho_{D E}-3 \zeta H\right)
$$

where $H, \zeta, \rho_{D M}, \rho_{D E}$ and $\omega$ are the Hubble parameter, bulk viscous coefficient, dark matter and energy density and equation of state ( $E O S)$ effective parameter respectively. To analyze the dynamical behavior of the above system, we introduce the following dimensionless variables:

$$
x=\rho_{D E} / 3 H^{2}, \quad y=1 /(\zeta / H+1)
$$

and obtain the autonomous ordinary differential equations (ODE):

$$
x^{\prime}=3(\mathrm{x}-1)(\omega x-1 / y+1)
$$

and

$$
y^{\prime}=\frac{3}{2} \mathrm{y}(\mathrm{y}-1)(2+\omega x-1 / y)
$$

where the prime denotes the derivative with respect to the e-folding number $N=\ln a$ ( $a$ is the cosmological scale factor). Three equilibrium critical points $P_{1}(0,1), P_{2}(1,1)$ and $P_{3}(1, /(2+$ $\omega)$ ) are found. The first one $P_{1}$ corresponds to the dark matter-dominated solution. In this case, 
the deceleration and effective $E o S$ parameters are $q=1 / 2$ and $\omega_{\text {eff }}=0$ respectively. Thus, we have a decelerated expansion. The related eigenvalues of the obtained linearized Jacobi stability matrix (LJSME) for the above system are $\mu_{1}=3 / 2$ and $\mu_{2}=-3 \omega$ and both have positive signs either we are in the quintessence or phantom case. Therefore, $P_{1}$ is unstable and this phase of a conventional dark matter dominance is needed for the observed cosmic structure formation. The second critical point $P_{2}$ corresponds either to no viscous dark energy-dominated solution if $\zeta=0$, or viscous dark energy-dominated solution if $\mathrm{H} \rightarrow \infty$. In this case, the deceleration and effective EoS parameters are given by: $q=(1+3 \omega) / 2$ and $\omega_{\text {eff }}=\omega$ respectively. This solution corresponds to an accelerated expansion. The LJSME are $\mu_{1}=$ $3(1+\omega) / 2$ and $\mu_{2}=3 \omega$. Notice that for a quintessence dark energy $(-1<\omega<-1 / 3)$, this critical point has eigenvalues with opposite signs indicating that it is an unstable saddle point. However, for a phantom dark energy $(\omega<-1)$, it has negative eigenvalues. Then, $P_{2}$ is stable corresponding to a big rip singularity attractor. Contrary to ref.[14], one can shown that if we assume the bulk viscosity as a linear combination of the two terms; one is constant, and the other is proportional to the scalar expansion $\theta=3 \mathrm{H}$ and by a proper choice of the parameters, the big rip problem can be prevented. The third critical point $P_{3}$ exists just for the quintessence dark energy scenario and it is not a physical solution in the case of the phantom case. It is of De Sitter viscous dark energy-dominated solution type characterized by $\zeta=H(1+$ $\omega), q=-1$ and $\omega_{\text {eff }}=-1$. In this case the cosmic expansion is accelerating, due to the presence of the viscosity. The LJSME are $\mu_{1}=-3$ and $\mu_{2}=-3(1+\omega) / 2$. We have found that it is stable because it has negative eigenvalues and regardless of the initial conditions for both cases, the universe will enter a final state dominated by dark energy. Figs.(1) and (2) display the portrait phase plane diagrams for the classical SC model in the phantom and quintessence cases with $\omega=-10 / 3$ and $\omega=-3 / 4$ respectively.

Now, due to the loop quantum effect, the modified flat space Friedmann equations can be written as [11]:

$$
H^{2}=\rho\left(1-\rho / \rho_{c}\right) / 3
$$

and

$$
\dot{H}=-\left(\rho_{D M}+(1+\omega) \rho_{D E}-3 \zeta H\right)\left(1-2 \rho / \rho_{c}\right) / 2
$$

where $\rho_{c} \equiv \sqrt{3} /\left(16 \pi^{2} \gamma^{3} G^{2} h\right)$ is the critical density in LQG, $\gamma$ the Barbero Immirzi parameter where its value is determined by the black hole entropy calculation, $h$ and $G$ are the Planck and Newton gravitational constants respectively. To make a dynamical analyzis, one has to add another new dynamical variable $\mathrm{z}=\rho / \rho_{c}$ to obtain the following autonomous system of ODE:

and

$$
\begin{gathered}
x^{\prime}=3(x(1-2 z)-1)(\omega x y-1+y) / y-3 x z /(1-z) \\
y^{\prime}=\frac{3}{2}(y-1)(y /(1-z)+\omega x y-1+y)(1-2 z)
\end{gathered}
$$

$$
z^{\prime}=-3 z-3 z(1-z)(\omega x y-1+y) / y
$$

For the physical solutions where $0<\mathrm{x}<1,0<\mathrm{y}<1$ and $0<\mathrm{z}<1$, we obtain the same critical points as in SC but their stability properties are different. The equilibrium critical points are: $P_{1}(0,1,0), P_{2}(1,1,0)$ and $P_{3}(1,1 /(2+\omega), 0)$. We have found that $P_{1}$ (the dark matter-dominated solution) becomes a saddle point (it was unstable in the SC case). The three LJSME are $\mu_{1}=3 / 2, \mu_{2}=-3 \omega$ and $\mu_{3}=-3$ and have different signs, but still this point is unstable for both phantom and quintessence dark energy. As it is in the SC case, we have a decelerated expansion. For the critical point $P_{2}$ (viscous dark energy-dominated solution) the LJSME are $\mu_{1}=3(1+\omega) / 2, \mu_{2}=3 \omega$ and $\mu_{3}=-3(1+\omega)$ Its stability for the phantom scenario $(\omega<-1)$ is different, from the SC case. In fact, this point is converted from a stable to a 
saddle point because it possesses eigenvalues with opposite signs. Hence, for the phantom scenario case, the quantum correction effect breaks the stability of the point $P_{2}$ and therefore, the problem of the future singularity (big rip) does not occur. For the quintessence case, this point remains a saddle point. For both cases, we have near the vicinity of this point an accelerated expansion. For the critical point $P_{3}$ which is of De Sitter type ( viscous dark energy-dominated solution), it exists just for the quintessence scenario and the LJSME are : $\mu_{1}=-3, \mu_{2}=-3(1+\omega) / 2$ and $\mu_{3}=0$. We have also an accelerated expansion. Notice that since the Jacobian matrix has one of the eigenvalues zero and the others are negative, the critical point is not hyperbolic. Thus, we cannot guaranty its stability properties directly from the eigenvalues. Instead, we have either to find the Lyapunov's functions which is in general a very hard task to do and there is no systematic way to find and construct these functions satisfying a number of conditions or to use the center manifold theorem (as it is in our dynamical study) which allows us to simplify the dynamical system by reducing its dimensionality.

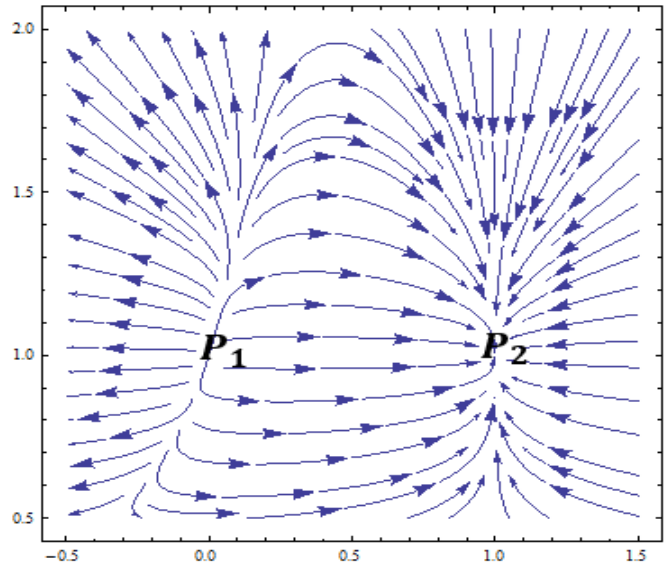

FIG.1: Phase-plane diagram for SC model in the phantom scenario with $\omega=-10 / 3$

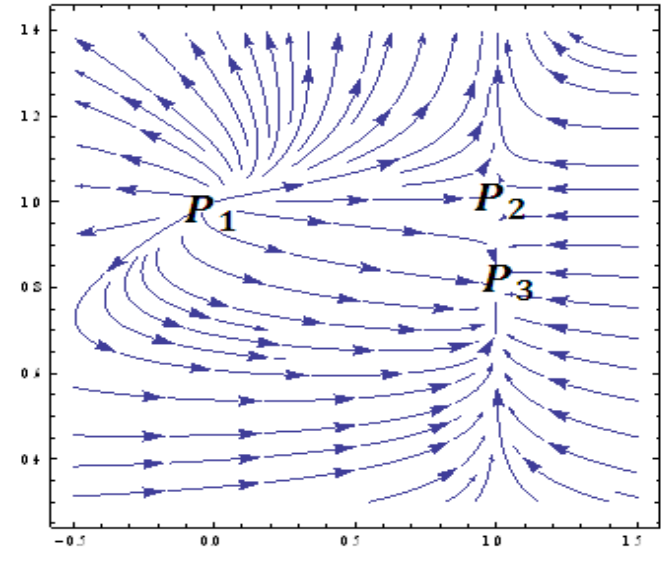

FIG.2: Phase-plane diagram for SC model in the quintessence scenario with $\omega=-3 / 4$

\section{Conclusion:}

We have studied in the context of both classical SC and LQC a dynamical system of a pressureless DM and viscous phantom or quintessence DE. The viscosity coefficient is taken to be a constant. We have found that in SC for phantom dark energy $(\omega<-1)$, there are two critical points one is unstable dark matter dominated solution, and the other one corresponds to a big rip singularity solution whereas for quintessence $(-1<\omega<-1 / 3)$, there are three critical points, two of them are unstable and the other one is stable corresponding to a De Sitter viscous dark energy. That is, the bulk viscosity has an effect only on quintessence model, which leads to the existence of an interesting viscous late time attractor stable solution of De Sitter type. However, by including the quantum geometry effects, we have obtained the same critical points but with almost different stability properties. In fact, the dark matter-dominated solution for both phantom and quintessence models which was unstable in the SC case becomes a saddle point in LQC. Thus, if we take into account the bulk viscosity and quantum geometry effects together in the evolution of the universe one has: for the quintessence model our universe will enter an era dominated by a viscous dark energy and accelerate forever, and for the phantom case the universe will enter an oscillatory regime (more study are under investigation). 


\section{References}

[1] S. Tsujikawa, Dark energy: investigation and modeling, invited review chapter on dark energy for a book "Dark Matter and Dark Energy: a Challenge for the 21st Century",[astro-ph./ 1004.1493

[2] W. M. Wood-Vasey et al. Astrophys. J..666 (2007) 694[astro-ph/0701041].

[3] Paul J. Steinhardt, Phil. Trans. R. Soc. Lond. A 361 (2003),2497.

[4] H.Aissaoui, N.Mebarki and H.Bouhalouf, FRW Like Cosmological Model and Accelerated Expansion of the Universe from Non Commutative Seiberg-Witten Geometry, in proceedings of Third Algerian Workshop On Astronomy and Astrophysics, 12-13 June 2010, Constantine, Algeria, AIP Conf.Proc. 1295 (2010) 164.

[5] A.G. Riess, et al., Astron. J. 116 (1998) 1009 , J. L. Tonry et al, Astrophys. J. 594, (2003)1, [astro-ph/0305008]

[6] C.Bennett et al., ApJS, 148 (2003) 1

[7] M.Tegmark et al. , Phys. Rev. D, 69 (2004) 103501

[8] Nojiri, S., Odintsov, S. D.: Phys. Lett. B 562 (2003)147.

[9] A.Ashtekar and P. Singh, Class. Quant. Grav. 28 (2011) 213001,[gr-qc/1108.0893]

[10] M. Bojowald, Living Rev. Rel. 11 (2008),4

[11] A.Ashtekar,T.Pawlowski and P. Singh, Phys.Rev.D.74 (2006) 084003, [grqc/0607039]

[12]K. Xiao and J.Y. Zhu, Int.J.Mod.Phys.A25 (2010) 4993, [gr-qc/1006.5377]

[13]D.Samart and B. Gumjudpai, Phys.Rev.D76 (2007) 043514, [gr-qc/0704.3414]

[14] X.-H. Meng, J. Ren, and M.-G. Hu, Comun..Theor. Phys., 47 (2007) 379 